TRANSACTIONS OF THE

AMERICAN MATHEMATICAL SOCIETY

Volume 364, Number 2, February 2012, Pages 689-702

S 0002-9947(2011)05405-X

Article electronically published on September 29,201

\title{
STRICHARTZ ESTIMATES ON KERR BLACK HOLE BACKGROUNDS
}

\author{
MIHAI TOHANEANU
}

\begin{abstract}
We study the dispersive properties for the wave equation in the Kerr space-time with small angular momentum. The main result of this paper is to establish Strichartz estimates for solutions of the aforementioned equation. This follows a local energy decay result for the Kerr space-time obtained in earlier work of Tataru and the author, and uses the techniques and results by the author and collaborators (2010). As an application, we then prove global well-posedness and uniqueness for the energy critical semilinear wave equation with small initial data.
\end{abstract}

\section{INTRODUCTION}

Understanding the decay properties of solutions to the linear wave equation on Kerr backgrounds is considered a crucial first step in proving the stability of the Kerr solution to the Einstein equations. Until recently even the problem of obtaining uniform bounds for such solutions was completely open, and only some partial results (pointwise decay and energy bounds for azimuthal solutions away from the event horizon) were obtained in [6], 7]. Recently in [23] and independently in [4, [5], 1] uniform pointwise bounds as well as local energy decay were established for small angular momentum. The aim of this paper is to prove Strichartz estimates under the same assumption of small angular momentum.

The Kerr geometry in Boyer-Lindquist coordinates is given by

$$
d s^{2}=g_{t t} d t^{2}+g_{t \phi} d t d \phi+g_{r r} d r^{2}+g_{\phi \phi} d \phi^{2}+g_{\theta \theta} d \theta^{2},
$$

where $t \in \mathbb{R}, r>0,(\phi, \theta)$ are the spherical coordinates on $\mathbb{S}^{2}$ and

$$
\begin{gathered}
g_{t t}=-\frac{\Delta-a^{2} \sin ^{2} \theta}{\rho^{2}}, \quad g_{t \phi}=-2 a \frac{2 M r \sin ^{2} \theta}{\rho^{2}}, \quad g_{r r}=\frac{\rho^{2}}{\Delta} \\
g_{\phi \phi}=\frac{\left(r^{2}+a^{2}\right)^{2}-a^{2} \Delta \sin ^{2} \theta}{\rho^{2}} \sin ^{2} \theta, \quad g_{\theta \theta}=\rho^{2}
\end{gathered}
$$

with

$$
\Delta=r^{2}-2 M r+a^{2}, \quad \rho^{2}=r^{2}+a^{2} \cos ^{2} \theta .
$$

Here $M$ represents the mass of the black hole and $a M$ its angular momentum.

Received by the editors January 8, 2010.

2010 Mathematics Subject Classification. Primary 35Q75.

(C)2011 American Mathematical Society Reverts to public domain 28 years from publication 
A straightforward computation gives us the inverse of the metric:

$$
\begin{gathered}
g^{t t}=-\frac{\left(r^{2}+a^{2}\right)^{2}-a^{2} \Delta \sin ^{2} \theta}{\rho^{2} \Delta}, \quad g^{t \phi}=-a \frac{2 M r}{\rho^{2} \Delta}, \quad g^{r r}=\frac{\Delta}{\rho^{2}}, \\
g^{\phi \phi}=\frac{\Delta-a^{2} \sin ^{2} \theta}{\rho^{2} \Delta \sin ^{2} \theta}, \quad g^{\theta \theta}=\frac{1}{\rho^{2}} .
\end{gathered}
$$

The case $a=0$ corresponds to the Schwarzschild space-time. We shall subsequently assume that $a$ is small $a \ll M$, so that the Kerr metric is a small perturbation of the Schwarzschild metric. We let $\square_{\mathbf{K}}$ denote the d'Alembertian associated to the Kerr metric.

In the above coordinates the Kerr metric has singularities at $r=0$ on the equator $\theta=\pi / 2$ and at the roots of $\Delta$, namely $r_{ \pm}=M \pm \sqrt{M^{2}-a^{2}}$. The singularity at $r=r_{+}$is just a coordinate singularity and corresponds to the event horizon. The singularity at $r=r_{-}$is also a coordinate singularity; for a further discussion of its nature, which is not relevant for our results, we refer the reader to [3, 9. To remove the singularities at $r=r_{ \pm}$we introduce functions $r^{*}, v_{+}$and $\phi_{+}$so that (see [9])

$$
d r^{*}=\left(r^{2}+a^{2}\right) \Delta^{-1} d r, \quad d v_{+}=d t+d r^{*}, \quad d \phi_{+}=d \phi+a \Delta^{-1} d r .
$$

The metric then becomes

$$
\begin{aligned}
d s^{2}= & -\left(1-\frac{2 M r}{\rho^{2}}\right) d v_{+}^{2}+2 d r d v_{+}-4 a \rho^{-2} M r \sin ^{2} \theta d v_{+} d \phi_{+}-2 a \sin ^{2} \theta d r d \phi_{+}+\rho^{2} d \theta^{2} \\
& +\rho^{-2}\left[\left(r^{2}+a^{2}\right)^{2}-\Delta a^{2} \sin ^{2} \theta\right] \sin ^{2} \theta d \phi_{+}^{2}
\end{aligned}
$$

which is smooth and nondegenerate across the event horizon up to, but not including, $r=0$. Just as in [13] and 23], we introduce the function

$$
\tilde{v}=v_{+}-\mu(r),
$$

where $\mu$ is a smooth function of $r$. In the $\left(\tilde{v}, r, \phi_{+}, \theta\right)$ coordinates the metric has the form

$$
\begin{aligned}
d s^{2}= & \left(1-\frac{2 M r}{\rho^{2}}\right) d \tilde{v}^{2}+2\left(1-\left(1-\frac{2 M r}{\rho^{2}}\right) \mu^{\prime}(r)\right) d \tilde{v} d r \\
& -4 a \rho^{-2} M r \sin ^{2} \theta d \tilde{v} d \phi_{+}+\left(2 \mu^{\prime}(r)-\left(1-\frac{2 M r}{\rho^{2}}\right)\left(\mu^{\prime}(r)\right)^{2}\right) d r^{2} \\
& -2 a \theta\left(1+2 \rho^{-2} M r \mu^{\prime}(r)\right) \sin ^{2} d r d \phi_{+}+\rho^{2} d \theta^{2} \\
& +\rho^{-2}\left[\left(r^{2}+a^{2}\right)^{2}-\Delta a^{2} \sin ^{2} \theta\right] \sin ^{2} \theta d \phi_{+}^{2} .
\end{aligned}
$$

On the function $\mu$ we impose the following two conditions:

(i) $\mu(r) \geq r^{*}$ for $r>2 M$, with equality for $r>5 M / 2$.

(ii) The surfaces $\tilde{v}=$ const are space-like, i.e.

$$
\mu^{\prime}(r)>0, \quad 2-\left(1-\frac{2 M r}{\rho^{2}}\right) \mu^{\prime}(r)>0 .
$$

As long as $a$ is small, we can work with the same function $\mu$ as in the case of the Schwarzschild space-time.

For convenience we also introduce

$$
\tilde{\phi}=\zeta(r) \phi_{+}+(1-\zeta(r)) \phi,
$$


where $\zeta$ is a cutoff function supported near the event horizon and work in the $(\tilde{v}, r, \tilde{\phi}, \theta)$ coordinates which are identical to $(t, r, \phi, \theta)$ outside of a small neighborhood of the event horizon.

Given $r_{-}<r_{e}<2 M$ we consider the wave equation

$$
\square_{\mathbf{K}} u=f
$$

in the cylindrical region

$$
\mathcal{M}_{R}=\left\{\tilde{v} \geq 0, r \geq r_{e}\right\}
$$

with initial data on the space-like surface

$$
\Sigma_{R}^{-}=\mathcal{M}_{R} \cap\{\tilde{v}=0\} .
$$

The lateral boundary of $\mathcal{M}_{R}$,

$$
\Sigma_{R}^{+}=\mathcal{M}_{R} \cap\left\{r=r_{e}\right\},
$$

is also space-like and can be thought of as the exit surface for all waves which cross the event horizon.

We define the initial (incoming) energy on $\Sigma_{R}^{-}$as

$$
E[u]\left(\Sigma_{R}^{-}\right)=\int_{\Sigma_{R}^{-}}\left(\left|\partial_{r} u\right|^{2}+\left|\partial_{\tilde{v}} u\right|^{2}+|\not \nabla u|^{2}\right) r^{2} d r d \omega,
$$

the outgoing energy on $\Sigma_{R}^{+}$as

$$
E[u]\left(\Sigma_{R}^{+}\right)=\int_{\Sigma_{R}^{+}}\left(\left|\partial_{r} u\right|^{2}+\left|\partial_{\tilde{v}} u\right|^{2}+|\not \nabla u|^{2}\right) r_{e}^{2} d \tilde{v} d \omega,
$$

and the energy on an arbitrary $\tilde{v}$ slice as

$$
E[u]\left(\tilde{v}_{0}\right)=\int_{\mathcal{M}_{R} \cap\left\{\tilde{v}=\tilde{v}_{0}\right\}}\left(\left|\partial_{r} u\right|^{2}+\left|\partial_{\tilde{v}} u\right|^{2}+|\not \nabla u|^{2}\right) r^{2} d r d \omega .
$$

For solutions to the constant coefficient wave equation on $\mathbb{R} \times \mathbb{R}^{3}$, the well-known Strichartz estimates state that

$$
\left\|\left|D_{x}\right|^{-\rho_{1}} \nabla u\right\|_{L_{t}^{p_{1}} L_{x}^{q_{1}}} \lesssim\|\nabla u(0)\|_{L^{2}}+\left\|\left|D_{x}\right|^{\rho_{2}} f\right\|_{L_{t}^{p^{\prime}} L_{x}^{q_{2}^{\prime}}} .
$$

Here the exponents $\left(\rho_{i}, p_{i}, q_{i}\right)$ are subject to the scaling relation

$$
\frac{1}{p}+\frac{3}{q}=\frac{3}{2}-\rho
$$

and the dispersion relation

$$
\frac{1}{p}+\frac{1}{q} \leq \frac{1}{2}, \quad 2<p \leq \infty .
$$

All pairs $(\rho, p, q)$ satisfying (1.9) and (1.10) are called Strichartz pairs. Those for which the equality holds in (1.10) are called sharp Strichartz pairs. Such estimates first appeared in the seminal works [2, 18, 19] and as stated include contributions from, e.g., 8, 16, 10, 12, and [11.

If one allows variable coefficients, such estimates are well understood locallyin-time. For smooth coefficients, this was first shown in [15] and later for $C^{2}$ coefficients in [17] and 20, 21, 22].

Globally-in-time, the problem is more delicate. Even a small, smooth, compactly supported perturbation of the flat metric may refocus a group of rays and produce caustics. Thus, constructing a parametrix for incoming rays proves to be quite 
difficult. At the same time, one needs to contend with the possibility of trapped rays at high frequencies and with eigenfunctions/resonances at low frequencies.

Our main theorem is the following global-in-time estimate:

Theorem 1.1. If $u$ solves $\square_{\mathbf{K}} u=f$ in $\mathcal{M}_{R}$, then for all nonsharp Strichartz pairs $\left(\rho_{1}, p_{1}, q_{1}\right)$ and $\left(\rho_{2}, p_{2}, q_{2}\right)$ we have

$$
E[u]\left(\Sigma_{R}^{+}\right)+\sup _{\tilde{v}} E[u](\tilde{v})+\|\nabla u\|_{L_{\tilde{v}}^{p_{1}} \dot{H}_{x}^{-\rho_{1}, q_{1}}}^{2} \lesssim E[u]\left(\Sigma_{R}^{-}\right)+\|f\|_{L_{\tilde{v}}^{p_{2}^{\prime}} \dot{H}_{x}^{\rho_{2}, q_{2}^{\prime}}}^{2} .
$$

Here the Sobolev-type spaces $\dot{H}^{s, p}$ coincide with the usual $\dot{H}^{s, p}$ homogeneous spaces in $\mathbb{R}^{3}$ expressed in polar coordinates $(r, \omega)$.

As a corollary of this result one can consider the global solvability question for the energy critical semilinear wave equation in the Kerr space. Let

$$
\Sigma_{0}=\{t=0\} .
$$

Note that $\Sigma_{0}$ is a smooth, space-like surface.

We now consider the Cauchy problem with initial data on $\Sigma_{0}$ :

$$
\begin{cases}\square_{\mathbf{K}} u= \pm u^{5} & \text { in } \mathcal{M}, \\ u=u_{0}, \tilde{T} u=u_{1} & \text { in } \Sigma_{0} .\end{cases}
$$

Here $\tilde{T}$ is a vector field that is smooth, everywhere time-like and equals $\partial_{\tilde{v}}$ on $\Sigma_{0}$ outside $\mathcal{M}_{C}$. Observe that we cannot use $\partial_{\tilde{v}}$ on all of $\Sigma_{0}$ since it becomes space-like inside the ergosphere (i.e. when $g_{t t}>0$ ).

Theorem 1.2. Let $r_{e}>r_{-}$. Then there exists $\epsilon>0$ so that for each initial data $\left(u_{0}, u_{1}\right)$ which satisfies

$$
E[u]\left(\Sigma_{0}\right) \leq \epsilon,
$$

the equation (1.12) admits a unique solution $u$ in the region $\left\{r>r_{e}\right\}$ which satisfies the bound

$$
E[u]\left(\Sigma_{r_{e}}\right)+\|u\|_{\dot{H}^{s, p}\left(\left\{r>r_{e}\right\}\right)} \lesssim E[u]\left(\Sigma_{0}\right)
$$

for all indices $s, p$ satisfying

$$
\frac{4}{p}=s+\frac{1}{2}, \quad 0 \leq s<\frac{1}{2} .
$$

Furthermore, the solution has a Lipschitz dependence on the initial data in the above topology.

Theorem 1.2 follows from Theorem 1.1 exactly as in Section 5 of [13]; hence we will now focus on proving the latter.

\section{The Strichartz estimates}

In this section we will prove Theorem 1.1. The key to the proof is the approach developed in 14 for small perturbations of the Minkowski metric and in 13, for the Schwarzschild metric which allows one to establish global-in-time Strichartz estimates provided that a strong form of the local energy estimates holds.

A weaker local energy result has been proved in [23]. We first improve this to a stronger result that only requires logarithmic losses in the energy norm. We then apply the techniques from [14] and 13] to obtain Strichartz estimates for all nonsharp exponents. 
Let us first recall the setup and results from [23. Let $\tau, \xi, \Phi$ and $\Theta$ be the Fourier variables corresponding to $t, r, \phi$ and $\theta$, and let

$$
p(t, r, \phi, \theta, \tau, \xi, \Phi, \Theta)=g^{t t} \tau^{2}+2 g^{t \phi} \tau \Phi+g^{\phi \phi} \Phi^{2}+g^{r r} \xi^{2}+g^{\theta \theta} \Theta^{2}
$$

be the principal symbol of $\square_{\mathbf{K}}$. On any null geodesic one has

$$
p(t, r, \phi, \theta, \tau, \xi, \Phi, \Theta)=0 \text {. }
$$

Moreover, all trapped null geodesics in the exterior $r>r_{+}$must also satisfy (see 23 for more details)

$$
\begin{gathered}
\xi=0, \\
r=\text { constant so that }\left(2 r \Delta-(r-M) \rho^{2}\right)^{2} \leq 4 a^{2} r^{2} \Delta \sin ^{2} \theta, \\
R_{a}(r, \tau, \Phi)=0,
\end{gathered}
$$

where

$$
\begin{aligned}
R_{a}(r, \tau, \Phi)= & \left(r^{2}+a^{2}\right)\left(r^{3}-3 M r^{2}+a^{2} r+a^{2} M\right) \tau^{2} \\
& -2 a M\left(r^{2}-a^{2}\right) \tau \Phi-a^{2}(r-M) \Phi^{2} .
\end{aligned}
$$

By (2.1) we can bound $\Phi$ in terms of $\tau$,

$$
|\Phi| \leq 4 M|\tau| \text {. }
$$

For $\Phi$ in this range and small $a$ the polynomial $\tau^{-2} R_{a}(r, \tau, \Phi)$ can be viewed as a small perturbation of

$$
\tau^{-2} R_{0}(r, \tau, \Phi)=r^{4}(r-3 M),
$$

which has a simple root at $r=3 M$. Hence for small $a$ the polynomial $R_{a}$ has a simple root close to $3 M$, which we denote by $r_{a}(\tau, \Phi)$.

In 23 the following result is proved:

Theorem 2.1. Let $u$ solve $\square_{\mathbf{K}} u=f$ in $\mathcal{M}_{R}$. Then

$$
\|u\|_{L E W_{\mathbf{K}}}^{2}+\sup _{\tilde{v}} E[u](\tilde{v})+E[u]\left(\Sigma_{R}^{+}\right) \lesssim E[u]\left(\Sigma_{R}^{-}\right)+\|f\|_{L E W_{K}^{*}}^{2} \cdot
$$

The exact definition of the norms $L E W_{\mathbf{K}}$ is not important. What matters is that it is equivalent to $H_{t, x}^{1}$ on compact sets outside an $O(1)$ neighborhood of $r=3 M$ and is degenerate on the trapped set (described above). Near infinity (say for $r>4 M)$ the norm $L E W_{\mathbf{K}}$ is defined on dyadic regions as follows:

$$
\|u\|_{L E W_{\mathbf{K}}}=\sup _{j \in \mathbb{Z}} 2^{-\frac{j}{2}}\left\|\nabla_{t, x} u\right\|_{L^{2}\left(\mathbb{R} \times\left\{|x| \in\left[2^{j-1}, 2^{j}\right]\right\}\right)}+2^{-\frac{3 j}{2}}\|u\|_{L^{2}\left(\mathbb{R} \times\left\{|x| \in\left[2^{j-1}, 2^{j}\right]\right\}\right)} .
$$

The norm $L E W_{\mathbf{K}}^{*}$ is the dual of $L E W_{\mathbf{K}}$. In particular, near infinity it will be defined as

$$
\|f\|_{L E W_{\mathbf{K}}^{*}}=\sum_{j \in \mathbb{Z}} 2^{\frac{j}{2}}\|f\|_{L^{2}\left(\mathbb{R} \times\left\{|x| \in\left[2^{j-1}, 2^{j}\right]\right\}\right)} .
$$

We first improve Theorem 2.1 around $r=3 M$. We will work with the usual Boyer-Lindquist coordinates $(t, r, \phi, \theta)$ since we are only interested in improving the estimate around $r=3 M$. In these coordinates we can write (2.9)

$$
\rho^{2} \sqrt{\Delta} \square_{\mathbf{K}} \frac{1}{\sqrt{\Delta}}=L_{K}=\Delta \partial_{r}^{2}+\left(-\frac{\left(r^{2}+a^{2}\right)^{2}}{\Delta} \partial_{t}^{2}-a \frac{2 M r}{\Delta} \partial_{t} \partial_{\phi}-\frac{a^{2}}{\Delta} \partial_{\phi}^{2}+L_{a}\right)+V(r),
$$


where

and

$$
L_{a}=a^{2} \sin ^{2} \theta \partial_{t}^{2}+\frac{1}{\sin ^{2} \theta} \partial_{\phi}^{2}+\frac{1}{\sin \theta} \partial_{\theta}\left(\sin \theta \partial_{\theta}\right)
$$

$$
V(r)=\sqrt{\Delta} \partial_{r}\left(\Delta \partial_{r} \frac{1}{\sqrt{\Delta}}\right) .
$$

We can now take the Fourier transform in $t$ (this is allowed since by Theorem $2.1 u$ is a tempered distribution in $t$ ) and $\phi$, and expand $\mathcal{F}_{t} L_{a}$ (which is an elliptic operator on $\mathbb{S}^{2}$ ) as the countable sum of its eigenvectors and corresponding eigenvalues $\lambda_{a}^{2}$ (note that $\lambda_{0}^{2}$ correspond to the usual spherical harmonics). This is possible since the operators $\partial_{\phi}$ and $L_{a}$ commute. We are left with the ordinary differential equation

$$
\left(\Delta \partial_{r}^{2}+V_{\lambda_{a}, \tau, \Phi}(r)\right) w(r)=g
$$

where

$$
V_{\lambda_{a}, \tau, \Phi}(r)=\tau^{2} W\left(r-r_{a}(\tau, \Phi)\right)-\lambda_{a}^{2}+V
$$

and $W(0)=W^{\prime}(0)=0, W^{\prime \prime}(0)>0$.

Let $\gamma_{0}: \mathbb{R} \rightarrow \mathbb{R}^{+}$be a smooth increasing function so that

$$
\gamma_{0}(y)= \begin{cases}1, & y<1 \\ y, & y \geq 2\end{cases}
$$

Let $\gamma_{1}: \mathbb{R}^{+} \rightarrow \mathbb{R}^{+}$be a smooth increasing function so that

$$
\gamma_{1}(y)=\left\{\begin{array}{cc}
y^{\frac{1}{2}}, \quad y<1 / 2 \\
1, \quad y \geq 1 .
\end{array}\right.
$$

Let $\gamma: \mathbb{R}^{2} \rightarrow \mathbb{R}^{+}$be a smooth function with the following properties:

$$
\gamma(y, z)=\left\{\begin{array}{cc}
1, & z<C, \\
\gamma_{0}(y), & y<\sqrt{z / 2}, z \geq C, \\
z^{\frac{1}{2}} \gamma_{1}\left(y^{2} / z\right), & y \geq \sqrt{z / 2}, z \geq C,
\end{array}\right.
$$

where $C$ is a large constant. In the sequel $z$ is either a discrete parameter or very large (see Remark 2.2), so the lack of smoothness at $z=C$ is of no consequence.

We define the symbol

$$
b_{p s}\left(r, \tau, \xi, \Phi, \lambda_{a}\right)=\gamma\left(-\psi\left(\frac{\lambda_{a}}{\tau}\right) \ln \left(\left(r-r_{a}(\tau, \Phi)\right)^{2}+\lambda_{a}^{-2} \xi^{2}\right), \ln \lambda_{a}\right)
$$

and its inverse

$$
b_{p s}^{-1}\left(r, \tau, \xi, \Phi, \lambda_{a}\right)=\frac{1}{\gamma\left(-\psi\left(\frac{\lambda_{a}}{\tau}\right) \ln \left(\left(r-r_{a}(\tau, \Phi)\right)^{2}+\lambda_{a}^{-2} \xi^{2}\right), \ln \lambda_{a}\right)} .
$$

Here $\psi: \mathbb{R} \rightarrow \mathbb{R}$ is a smooth cutoff such that

$$
\psi(y)=\left\{\begin{array}{lc}
1, & y \in\left[-C,-\frac{1}{C}\right] \cup\left[\frac{1}{C}, C\right], \\
0, & y \in(-\infty,-2 C] \cup\left[-\frac{1}{2 C}, \frac{1}{2 C}\right] \cup[2 C, \infty),
\end{array}\right.
$$

where $C$ is a large enough constant. The role of $\psi$ is to make sure that $b_{p s}=1$ when $\tau \ll \lambda_{a}$ and $\tau \gg \lambda_{a}$.

Observe that, as opposed to their Schwarzschild counterparts $a_{p s}$ and $a_{p s}^{-1}$ defined in [13], the symbols $b_{p s}$ and $b_{p s}^{-1}$ will depend on $\tau$ and $\Phi$. 
We note that if $\lambda_{a}$ is small, then $b_{p s}$ and $b_{p s}^{-1}$ both equal 1 , while if $\lambda_{a}$ is large, then they satisfy the bounds

$$
\begin{gathered}
1 \leq b_{p s}\left(r, \tau, \xi, \Phi, \lambda_{a}\right) \leq b_{p s}\left(r, \tau, 0, \Phi, \lambda_{a}\right) \leq\left(\ln \lambda_{a}\right)^{\frac{1}{2}}, \\
\left(\ln \lambda_{a}\right)^{-\frac{1}{2}} \leq b_{p s}^{-1}\left(r, \tau, 0, \Phi, \lambda_{a}\right) \leq b_{p s}^{-1}\left(r, \tau, \xi, \Phi, \lambda_{a}\right) \leq 1 .
\end{gathered}
$$

We also observe that the region where $y^{2}>z$ corresponds to $\left(r-r_{a}(\tau, \Phi)\right)^{2}+$ $\lambda_{a}^{-2} \xi^{2}<e^{-\sqrt{\ln \lambda_{a}}}$. Thus differentiating the two symbols we obtain the following bounds:

$$
\begin{array}{r}
\left|\partial_{r}^{\alpha} \partial_{\xi}^{\beta} \partial_{\lambda_{a}}^{\nu} \partial_{\tau}^{\eta} b_{p s}\left(r, \tau, \xi, \Phi, \lambda_{a}\right)\right| \leq c_{\alpha, \beta, \nu}\left(1+\left|\ln \left(\left(r-r_{a}(\tau, \Phi)\right)^{2}+\lambda_{a}^{-2} \xi^{2}\right)\right|\right) \lambda_{a}^{-\beta-\nu-\eta} \\
\times\left(\left(r-r_{a}(\tau, \Phi)\right)^{2}+\lambda_{a}^{-2} \xi^{2}+e^{-\sqrt{\ln \lambda_{a}}}\right)^{-\frac{\alpha+\beta+\eta}{2}}
\end{array}
$$

respectively,

$$
\begin{aligned}
\left|\partial_{r}^{\alpha} \partial_{\xi}^{\beta} \partial_{\lambda_{a}}^{\nu} \partial_{\tau}^{\eta} b_{p s}^{-1}\left(r, \tau, \xi, \Phi, \lambda_{a}\right)\right| & \leq c_{\alpha, \beta, \nu} b_{p s}^{-2}\left(r, \tau, \xi, \Phi, \lambda_{a}\right)\left(1+\left|\ln \left(\left(r-r_{a}(\tau, \Phi)\right)^{2}+\lambda_{a}^{-2} \xi^{2}\right)\right|\right) \\
& \times \lambda_{a}^{-\beta-\nu-\eta}\left(\left(r-r_{a}(\tau, \Phi)\right)^{2}+\lambda_{a}^{-2} \xi^{2}+e^{-\sqrt{\ln \lambda_{a}}}\right)^{-\frac{\alpha+\beta+\eta}{2}}
\end{aligned}
$$

when $\alpha+\beta+\nu+\eta>0$ and $\ln \lambda \geq C$. These show that we have a good operator calculus for the corresponding pseudodifferential operators. In particular, in terms of the classical symbol classes we have

$$
b_{p s} \in S_{1-\delta, 0}^{\delta}, \quad b_{p s}^{-1} \in S_{1-\delta, 0}^{0}, \quad \delta>0 .
$$

By (2.14) and 2.15) one easily sees that these operators are approximate inverses. More precisely, for small $\lambda_{a}, \ln \lambda_{a}<C$, they are both the identity, while for large $\lambda_{a}$,

$$
\left\|b_{p s}^{w}\left(\lambda_{a}\right)\left(b_{p s}^{-1}\right)^{w}\left(\lambda_{a}\right)-I\right\|_{L^{2} \rightarrow L^{2}} \lesssim \lambda_{a}^{-1} e^{\sqrt{\ln \lambda_{a}}}, \quad \ln \lambda_{a} \geq C .
$$

Choosing $C$ large enough we ensure that the bound above is always much smaller than 1.

Remark 2.2. The role played by $\lambda_{a}$ changes from the proof of Theorem 2.3 to the proof of the Strichartz estimates. Since all of our $L^{2}$ estimates admit orthogonal decompositions with respect to the eigenfunctions associated to $\lambda_{a}$, it suffices for Theorem 2.3 to fix $\lambda_{a}$ and work with the operators $b_{p s}^{w}\left(\lambda_{a}\right)$. However, in the proof of the Strichartz estimates we need kernel bounds for operators of the form $B_{p s}$, which is why we think of $\lambda_{a}$ as a fourth Fourier variable (besides $\tau, \Phi$ and $\xi$ ) and track the symbol regularity with respect to $\lambda_{a}$ as well. Of course, this is meaningless for $\lambda_{a}$ in a compact set; only the asymptotic behavior as $\lambda_{a} \rightarrow \infty$ is relevant.

We can now introduce the Weyl operators

$$
B_{p s}=\sum_{\lambda_{a}} b_{p s}^{w}\left(\lambda_{a}\right) \Pi_{\lambda_{a}}
$$

respectively,

$$
B_{p s}^{-1}=\sum_{\lambda_{a}}\left(b_{p s}^{-1}\right)^{w}\left(\lambda_{a}\right) \Pi_{\lambda_{a}},
$$

where $\Pi_{\lambda_{a}}$ are the spectral projectors on the eigenspaces of $L_{a}$ determined by $\lambda_{a}$. We remark that $B_{p s} u$ and $B_{p s}^{-1} u$ are supported near $r=3 M$ if $u$ is also. Indeed, 
this is a simple consequence of the fact that $b_{p s}\left(\lambda_{a}\right) \equiv 1$ away from $r=3 M$. This will be used later in the proof of Proposition 2.8 .

We use these two operators in order to define the improved local energy norms around $r=3 M$. Let $I$ be a small neighborhood of $3 M$ (which does not depend on a). We say that $\tilde{u} \in L E K_{p s}$ if $\tilde{u}: \mathbb{R} \times I \times \mathbb{S}^{2} \rightarrow \mathbb{R}$ and

$$
\|\tilde{u}\|_{L E K_{p s}}=\left\|B_{p s}^{-1} \tilde{u}\right\|_{H^{1}} \approx\left\|B_{p s}^{-1} \nabla \tilde{u}\right\|_{L^{2}}<\infty .
$$

Similarly, $\tilde{f} \in L E K_{p s}^{*}$ if $\tilde{f}: \mathbb{R} \times I \times \mathbb{S}^{2} \rightarrow \mathbb{R}$ and

$$
\|\tilde{f}\|_{L E K_{p s}^{*}}=\left\|B_{p s} \tilde{f}\right\|_{L^{2}}<\infty .
$$

Let $\chi(r)$ be a cutoff function supported on $I$ which equals 1 on a smaller neighborhood of size $O(1)$ near $3 M$, and let $u: \mathcal{M}_{R} \rightarrow \mathbb{R}$. We say that $u \in L E_{\mathbf{K}}$ if $\chi u$ is the restriction of some $\tilde{u} \in L E K_{p s}$ on $\mathcal{M}_{R}$, and

$$
\|u\|_{L E_{\mathbf{K}}}=\inf _{\left.\tilde{u}\right|_{\mathcal{M}_{R}=\chi u}}\|\tilde{u}\|_{L E K_{p s}}+\|(1-\chi) u\|_{L E W_{\mathbf{K}}} .
$$

Similarly if $f: \mathcal{M}_{R} \rightarrow \mathbb{R}$, then $f \in L E_{\mathbf{K}}^{*}$ if $\chi f$ is the restriction of some $\tilde{f} \in L E K_{p s}^{*}$ on $\mathcal{M}_{R}$, and

$$
\|f\|_{L E_{\mathbf{K}}^{*}}=\inf _{\left.\tilde{f}\right|_{\mathcal{M}_{R}}=\chi f}\|\tilde{f}\|_{L E K_{p s}^{*}}+\|(1-\chi) f\|_{L E W_{\mathbf{K}}^{*}} .
$$

Our improved local energy estimate reads:

Theorem 2.3. For all functions $u$ which solve $\square_{\mathbf{K}} u=f$ in $\mathcal{M}_{R}$ we have

$$
\sup _{\tilde{v}>0} E[u](\tilde{v})+E[u]\left(\Sigma_{R}^{+}\right)+\|u\|_{L E_{\mathbf{K}}}^{2} \lesssim E[u]\left(\Sigma_{R}^{-}\right)+\|f\|_{L E_{\mathbf{K}}^{*}}^{2} .
$$

Proof. We start with the following estimate near $r=3 M$, which is the equivalent of Proposition 3.3 from [13:

Proposition 2.4. a) Let $u$ be a function supported in $\{5 M / 2<r<5 M\}$ which solves $\square_{\mathbf{K}} u=f$. Then

$$
\|u\|_{L E K_{p s}}^{2} \lesssim\|f\|_{L E K_{p s}^{*}}^{2} .
$$

b) Let $f \in L E K_{p s}{ }^{*}$ be supported in $\{11 M / 4<r<4 M\}$. Then there is a function $u$ supported in $\{5 M / 2<r<5 M\}$ so that

$$
\sup _{t} E[u]+\|u\|_{L E K_{p s}}^{2}+\left\|\square_{\mathbf{K}} u-f\right\|_{L E_{\mathbf{K}}^{*}}^{2} \lesssim\|f\|_{L E K_{p s}^{*}}^{2} .
$$

Proof. We start with part a). By Plancherel's formula and the fact that

$$
\Phi<\lambda_{a}
$$

(2.24) will follow if we can prove that

$$
\left\|\partial_{r} w(r)\right\|_{L^{2}}+\left(|\tau|+\left|\lambda_{a}\right|\right)\left\|\left(b_{p s}^{-1}\right)^{w}\left(\tau, \Phi, \lambda_{a}\right) w\right\|_{L^{2}} \lesssim\left\|b_{p s}^{w}\left(\tau, \Phi, \lambda_{a}\right) g\right\|_{L^{2}}
$$

for any fixed $\tau, \Phi$ and $\lambda_{a}$ (here $b_{p s}^{w}\left(\tau, \Phi, \lambda_{a}\right.$ ) is the one dimensional pseudodifferential operator obtained from $b_{p s}$ by fixing $\tau, \Phi$ and $\lambda_{a}$, and $w$ and $g$ satisfy (2.10).

Depending on the relative sizes of $\lambda_{a}, \tau$ and $\Phi$ and taking into account (2.26), we consider several cases. In the easier cases it suffices to replace the bound (2.27) with a simpler bound

$$
\left\|\partial_{r} w\right\|_{L^{2}}+\left(|\tau|+\left|\lambda_{a}\right|\right)\|w\|_{L^{2}} \lesssim\|g\|_{L^{2}} .
$$


Case $1\left(\lambda_{a}, \tau, \Phi<C\right)$. Then $V_{\lambda_{a}, \tau, \Phi}(r) \approx 1$. We solve (2.10) as a Cauchy problem with data on one side; namely, if

$$
E[w](r)=\Delta\left(\partial_{r} w\right)^{2}+w^{2},
$$

then an easy computation shows that

$$
\frac{d}{d r} E[w](r) \lesssim E[w](r)+g^{2}
$$

By Gronwall's inequality and the fact that $E[w](r)=0$ away from the photonsphere, we obtain the pointwise bound

$$
|w|+\left|\partial_{r} w\right| \lesssim\|g\|_{L^{2}}
$$

which easily implies (2.28).

Case $2\left(\Phi, \lambda_{a}<\frac{1}{C} \tau\right)$. Then $V_{\lambda_{a}, \tau, \Phi}(r) \approx \tau^{2}, \partial_{r} V_{\lambda_{a}, \tau, \Phi}(r) \approx \tau^{2}$ for $r$ in a compact set; therefore (2.10) is hyperbolic in nature. Hence we can solve (2.10) as a Cauchy problem with data on one side. Namely, if

$$
E[w](r)=\Delta\left(\partial_{r} w\right)^{2}+V_{\lambda_{a}, \tau, \Phi}(r) w^{2},
$$

then an easy computation shows that

$$
\frac{d}{d r} E[w](r) \lesssim E[w](r)+g^{2}
$$

By Gronwall's inequality and the fact that $E[w](r)=0$ away from the photonsphere, we obtain the pointwise bound

$$
\tau|w|+\left|\partial_{r} w\right| \lesssim\|g\|_{L^{2}}
$$

which implies (2.28).

Case $3\left(\lambda_{a}>C \tau\right)$. Then $V_{\lambda_{a}, \tau, \Phi}(r) \approx-\lambda_{a}^{2}$ for $r$ in a compact set; therefore (2.10) is elliptic. Then we solve (2.10) as an elliptic problem with Dirichlet boundary conditions on a compact interval and obtain

$$
\lambda_{a}^{\frac{3}{2}}\|w\|_{L^{2}}+\lambda_{a}^{\frac{1}{2}}\left\|\partial_{r} w\right\|_{L^{2}} \lesssim\|g\|_{L^{2}},
$$

which again gives (2.28).

Case 4. $\frac{1}{C} \lambda_{a}<\tau<C \lambda_{a}, C<\lambda_{a}, C<\tau$. In this case (2.28) no longer holds. However, we can now use Proposition 3.4 from [13] which states

Proposition 2.5. Let $W$ be a smooth function satisfying $W(0)=W^{\prime}(0)=0$, $W^{\prime \prime}(0)>0$, and $|\epsilon| \lesssim 1$. Let $w$ be a solution of the ordinary differential equation

$$
\left(\partial_{r^{*}}^{2}+\lambda^{2}\left(W\left(r^{*}\right)+\epsilon\right)\right) w\left(r^{*}\right)=g
$$

supported near $r^{*}=0$. Then we have

$$
\left\|\partial_{r^{*}} u\right\|_{L^{2}}+\lambda\left\|\left(a_{p s}^{-1}\right)^{w}(\lambda) u\right\|_{L^{2}} \lesssim\left\|a_{p s}^{w}(\lambda) g\right\|_{L^{2}} .
$$

Here the symbols $a_{p s}(\lambda)$ and $\left(a_{p s}^{-1}\right)(\lambda)$ are defined as follows:

$$
\begin{aligned}
a_{p s}(\lambda)\left(r^{*}, \xi\right) & =\gamma\left(-\ln \left(r^{* 2}+\lambda^{-2} \xi^{2}\right), \ln \lambda\right), \\
a_{p s}^{-1}(\lambda)\left(r^{*}, \xi\right) & =\frac{1}{\gamma\left(-\ln \left(r^{* 2}+\lambda^{-2} \xi^{2}\right), \ln \lambda\right)} .
\end{aligned}
$$


Since in this case $\psi\left(\frac{\lambda_{a}}{\tau}\right)=1$, we can apply Proposition 2.5 with $r^{*}=r-r_{a}(\tau, \Phi)$, $W$ as in (2.11) and with $b_{p s}^{w}\left(\tau, \Phi, \lambda_{a}\right)$ and $\left(b_{p s}^{-1}\right)^{w}\left(\tau, \Phi, \lambda_{a}\right)$ replacing $a_{p s}^{w}(\lambda)$ and $\left(a_{p s}^{-1}\right)^{w}(\lambda)$, respectively. Note that the extra $\Delta$ coefficient in front of $\partial_{r}^{2}$ in (2.10) plays no role, since $\Delta \approx \partial_{r} \Delta \approx 1$ near $r=3 M$.

Part b) follows now from part a) exactly as in Proposition 3.3 from [13] with $\lambda_{a}$ replacing the spherical harmonics $\lambda$ and $r^{*}=r-r_{a}(\tau, \Phi)$.

We now repeat the arguments in Section 4 of 13 to turn the improved local energy estimate, Proposition 2.4, into Strichartz estimates, Theorem 1.1. The only (minor) differences appear in proving Case II of Propositions 4.10 and 4.11, which we will settle below.

We thus need to prove

Proposition 2.6. For u supported in $\{5 M / 2<r<5 M\}$ we have

$$
\|\nabla u\|_{L_{t}^{p} H^{-\rho, q}}^{2} \lesssim E[u](0)+\|u\|_{L E K_{p s}}^{2}+\left\|\square_{K} u\right\|_{L E K_{p s}^{*}}^{2} .
$$

Proof. Clearly the operator $\square_{K}$ can be replaced by $L_{K}$. The potential $V$ can be neglected due to the straightforward bound

$$
\|V u\|_{L E_{p s}^{*}} \lesssim\|u\|_{L E_{p s}} .
$$

Indeed, for $u_{\lambda_{a}}$ the eigenfunction corresponding to the eigenvalue $\lambda_{a}^{2}$ we have

$$
\left\|V u_{\lambda_{a}}\right\|_{L E K_{p s}^{*}} \lesssim\left|\ln \left(2+\lambda_{a}\right)\right|^{\frac{1}{2}}\left\|u_{\lambda_{a}}\right\|_{L^{2}} \lesssim \lambda_{a}\left|\ln \left(2+\lambda_{a}\right)\right|^{-\frac{1}{2}}\left\|u_{\lambda_{a}}\right\|_{L^{2}} \lesssim\left\|u_{\lambda_{a}}\right\|_{L E_{p s}} .
$$

We introduce the auxiliary function

$$
\Psi=B_{p s}^{-1} u .
$$

By the definition of the $L E K_{p s}$ norm we have

$$
\|\Psi\|_{H^{1}} \lesssim\|u\|_{L E K_{p s}} .
$$

We also claim that

$$
\left\|L_{K} \Psi\right\|_{L^{2}} \lesssim\|u\|_{L E K_{p s}}+\left\|L_{K} u\right\|_{L^{2}} .
$$

Since $B_{p s}^{-1}$ is $L^{2}$ bounded, this is a consequence of the commutator bound

$$
\left[B_{p s}^{-1}, L_{K}\right]: L E K_{p s} \rightarrow L^{2}
$$

or equivalently

$$
\left[B_{p s}^{-1}, L_{K}\right] B_{p s}: H^{1} \rightarrow L^{2} .
$$

It suffices to consider the first term in the symbol calculus, as the remainder belongs to $O P S_{1-\delta, \delta}^{\delta}$, mapping $H^{\delta}$ to $L^{2}$ for all $\delta>0$. The symbol of the first term is $q\left(\tau, \Phi, \xi, r, \lambda_{a}\right)=\left\{b_{p s}^{-1}\left(\lambda_{a}\right), \Delta \xi^{2}-\frac{\left(r^{2}+a^{2}\right)^{2}}{\Delta} \tau^{2}-a \frac{2 M r}{\Delta} \tau \Phi-\frac{a^{2}}{\Delta} \Phi^{2}+\lambda_{a}^{2}\right\} b_{p s}\left(\lambda_{a}\right)$, and a priori we have $q \in S_{1-\delta, \delta}^{1+\delta}$. For a better estimate we compute the Poisson bracket

$$
\begin{aligned}
q\left(\tau, \Phi, \xi, r, \lambda_{a}\right)= & b_{p s}^{-1}\left(\lambda_{a}\right) \gamma_{y}\left(y, \ln \lambda_{a}\right) \psi\left(\frac{\Phi}{\tau}\right) \\
& \times \frac{4 \xi\left(r-r_{a}(\tau, \Phi)\right)-2 \xi \lambda_{a}^{-2} \tau^{2} W\left(r-r_{a}(\tau, \Phi)\right)+2(r-M) \xi^{2}}{\left(r-r_{a}(\tau, \Phi)\right)^{2}+\lambda_{a}^{-2} \xi^{2}},
\end{aligned}
$$


where $y=\left(r-r_{a}(\tau, \Phi)\right)^{2}+\lambda_{a}^{-2} \xi^{2}$ and $W \approx r^{2}$. The first three factors on the right are bounded. The fourth is bounded by $\max \left\{\lambda_{a}, \tau\right\}$ by Cauchy Schwarz and the fact that $q$ is supported in $|\xi| \lesssim \lambda_{a}$. Hence we obtain $q \in\left(\tau+\lambda_{a}\right) S_{1-\delta, \delta}^{0}$, and the commutator bound (2.32) follows.

Given (2.30) and (2.31), we localize $\Psi$ as in 13 to time intervals of unit length and then apply the local Strichartz estimates. By summing over these strips we obtain

$$
\|\nabla \Psi\|_{L^{p} H^{-\rho, q}} \lesssim\|u\|_{L E K_{p s}}+\left\|L_{K} u\right\|_{L^{2}}
$$

for all sharp Strichartz pairs $(\rho, p, q)$.

To return to $u$ we invert $B_{p s}^{-1}$,

$$
u=B_{p s} \Psi+\left(I-B_{p s} B_{p s}^{-1}\right) u .
$$

The second term is much more regular, since by (2.17) we have

$$
I-B_{p s} B_{p s}^{-1} \in S_{1-\delta, 0}^{-1+\delta}, \quad \delta>0 .
$$

This leads to

$$
\left\|\nabla\left(I-B_{p s} B_{p s}^{-1}\right) u\right\|_{L^{2} H^{1-\delta}} \lesssim\|u\|_{L E K_{p s}}, \quad \delta>0
$$

therefore all the Strichartz estimates are satisfied simply by Sobolev embeddings.

For the main term $B_{p s} \Psi$ we take advantage of the fact that we only seek to prove the nonsharp Strichartz estimates for $u$. The nonsharp Strichartz estimates for $\Psi$ are obtained from the sharp ones via Sobolev embeddings,

$$
\|\nabla \Psi\|_{H^{-\rho_{2}, q_{2}}} \lesssim\|\nabla \Psi\|_{H^{-\rho_{1}, q_{1}}}, \quad \frac{3}{q}_{2}+\rho_{2}=\frac{3}{q_{1}}+\rho_{1}, \quad \rho_{1}<\rho_{2} .
$$

To obtain the nonsharp estimates for $u$ instead, we need a slightly stronger form of the above bound, namely

Lemma 2.7. Assume that $\left(\rho_{1}, p_{1}, q_{1}\right),\left(\rho_{2}, p_{2}, q_{2}\right)$ are Strichartz pairs with $p_{1}<p_{2}$, $q_{1}<q_{2}<\infty$. Then

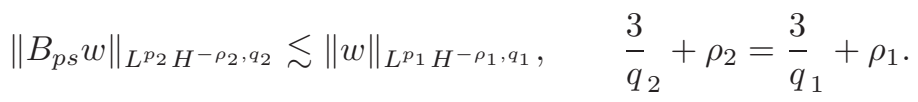

Proof. We need to prove that the operator

$$
\tilde{B}=O p^{w}\left(\xi^{2}+\lambda_{0}^{2}+1\right)^{-\frac{\rho_{2}}{2}} B_{p s} O p^{w}\left(\xi^{2}+\lambda_{0}^{2}+1\right)^{\frac{\rho_{1}}{2}}
$$

maps $L^{q_{1}}$ into $L^{q_{2}}$. The principal symbol of $\tilde{B}$ is

$$
\tilde{b}\left(r, \tau, \xi, \Phi, \lambda_{a}\right)=\left(\xi^{2}+\lambda_{0}^{2}+1\right)^{\frac{\rho_{1}-\rho_{2}}{2}} b_{p s}\left(r, \xi, \lambda_{a}\right),
$$

and by the pdo calculus the remainder is easy to estimate:

$$
\tilde{B}-\tilde{b}^{w} \in O P S_{1-\delta, 0}^{\rho_{1}-\rho_{2}-1+\delta}, \quad \delta>0 .
$$

The conclusion of the lemma will follow from the Hardy-Littlewood-Sobolev inequality (applied separately for $t, r$ and $\omega$ ) if we prove a suitable pointwise bound on the kernel $K$ of $b_{0}^{w}$, namely

$$
\left|K\left(t_{1}, r_{1}, \omega_{1}, t_{2}, r_{2}, \omega_{2}\right)\right| \lesssim\left|t_{1}-t_{2}\right|^{-1+\frac{1}{p_{1}}-\frac{1}{p_{2}}}\left(\left|r_{1}-r_{2}\right|\left|\omega_{1}-\omega_{2}\right|^{2}\right)^{-1+\frac{1}{q_{1}}-\frac{1}{q_{2}}}
$$

For fixed $r$ we consider a smooth dyadic partition of unity in frequency as follows:

$$
1=\left(1-\chi_{\left\{\tau \approx \lambda_{a}\right\}}\right) \chi_{\left\{|\xi|>\lambda_{a}\right\}} \chi_{\{\mu<1\}}+\chi_{\left\{\tau \approx \lambda_{a}\right\}} \sum_{\mu \geq 1 \text { dyadic }} \chi_{\left\{\lambda_{a} \approx \mu\right\}} \sum_{\nu=0}^{\mu} \chi_{\{|\xi| \approx \nu\}} .
$$


This leads to a similar decomposition for $\tilde{b}$, namely

$$
\tilde{b}=\tilde{b}_{00}+\sum_{\mu \geq 1} \sum_{\nu=0}^{\mu} \tilde{b}_{\mu \nu} .
$$

In the region $\{|\xi| \gtrsim \lambda\} \cup\{\mu<1\} \cup\{\tau \gg \lambda\} \cup\{\tau \ll \lambda\}$ the symbol $\tilde{b}$ is of class $S^{\rho_{1}-\rho_{2}}$, which yields a kernel bound for $\tilde{b}_{00}$ of the form

$$
\left|K_{00}\left(t_{1}, r_{1}, \omega_{1}, t_{2}, r_{2}, \omega_{2}\right)\right| \lesssim\left(\left|t_{1}-t_{2}\right|+\left|r_{1}-r_{2}\right|+\left|\omega_{1}-\omega_{2}\right|\right)^{-3+\rho_{2}-\rho_{1}} .
$$

The symbols of $\tilde{b}_{\mu \nu}$ are supported in $\{|\xi| \approx \nu, \lambda \approx \tau \approx \mu\}$, are smooth on the same scale and have size at most $\ln \left(\nu^{-1} \mu\right) \mu^{\rho_{1}-\rho_{2}}$. Clearly, on their support we also have $\lambda_{0} \approx \lambda_{a} \approx \mu$. Hence after integration by parts we get that their kernels satisfy bounds of the form

$$
\begin{aligned}
& \left|K_{\mu, \nu}\left(t_{1}, r_{1}, \omega_{1}, t_{2}, r_{2}, \omega_{2}\right)\right| \\
\lesssim & \ln \left(\nu^{-1} \mu\right) \mu^{\rho_{1}-\rho_{2}} \nu\left(\left|r_{1}-r_{2}\right| \nu+1\right)^{-N} \mu^{2}\left(\left|\omega_{1}-\omega_{2}\right| \mu+1\right)^{-N}\left(\left|t_{1}-t_{2}\right| \mu+1\right)^{-N} \\
\lesssim & \left|t_{1}-t_{2}\right|^{-1+\frac{1}{p_{1}}-\frac{1}{p_{2}}} \ln \left(\nu^{-1} \mu\right) \mu^{\frac{3}{q_{1}}-\frac{3}{q_{2}}} \nu\left(\left|r_{1}-r_{2}\right| \nu+1\right)^{-N} \mu^{2}\left(\left|\omega_{1}-\omega_{2}\right| \mu+1\right)^{-N}
\end{aligned}
$$

for all nonnegative integers $N$. Then (2.34) follows after summation.

Finally, we need to prove the following:

Proposition 2.8. There is a parametrix $P_{p s}$ for $\square_{\mathbf{K}}$ so that, given $f$ supported near $r=3 M, P_{p s}$ is also supported near $3 M$, and we have

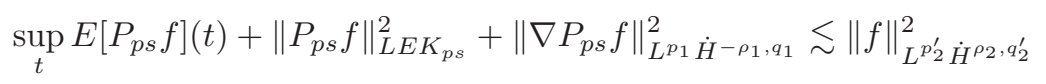

and the error estimate

$$
\left\|\square_{\mathbf{K}} P_{p s} f-f\right\|_{L E K_{p s}^{*}+L^{1} L^{2}} \lesssim\|f\|_{L^{p_{2}^{\prime}} \dot{H}^{\rho_{2}, q_{2}^{\prime}}}
$$

for all nonsharp Strichartz pairs $\left(\rho_{1}, p_{1}, q_{1}\right)$ and $\left(\rho_{2}, p_{2}, q_{2}\right)$.

Proof. By using local-in-time Strichartz estimates, one can produce (see 13]) a parametrix $\tilde{P}_{p s}$ so that $\tilde{P}_{p s} f$ is supported near $r=3 M$ and satisfies the bounds

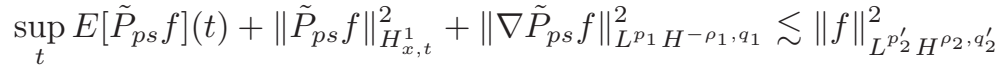

and the error estimate

$$
\left\|L_{K} \tilde{P}_{p s} f-f\right\|_{L_{x, t}^{2}} \lesssim\|f\|_{L^{p_{2}^{\prime} H^{\rho_{2}, q_{2}^{\prime}}}}
$$

We now define the localized parametrix near the photon sphere $P_{p s}$ as

$$
P_{p s} f=B_{p s}^{-1} \tilde{P}_{p s} B_{p s} f \text {. }
$$

By the remark after (2.19), $P_{p s} f$ is supported near $r=3 M$, so we need to show that it satisfies the required bounds.

By (2.33) we get

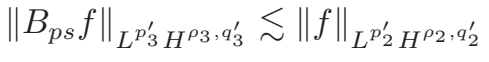

for some other nonsharp Strichartz pair $\left(\rho_{3}, p_{3}, q_{3}\right)$ with $p_{3}<p_{2}$ and $q_{3}<q_{2}$. Since $B_{p s}^{-1}$ is $L^{2}$ bounded, from the above bounds for $\tilde{K}_{p s}$ we obtain a part of (2.37), namely

$$
\left\|P_{p s} f\right\|_{L E K_{p s}}^{2} \lesssim\left\|\chi_{p s} f\right\|_{L^{p_{2}^{\prime} H^{\rho_{2}, q_{2}^{\prime}}}}^{2}
$$


The $L^{p_{1}} H^{-\rho_{1}, q_{1}}$ bound in (2.37) follows from (2.33) applied to the weaker operator $B_{p s}^{-1}$ instead of $B_{p s}$ :

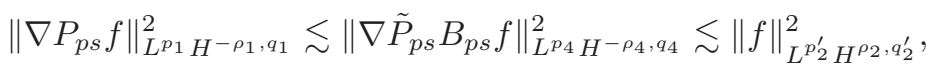

where $\left(\rho_{4}, p_{4}, q_{4}\right)$ is another Strichartz pair with $p_{4}<p_{1}$ and $q_{4}<q_{1}$.

To finish proving (2.35) we need to bound $E\left[P_{p s} f\right](t)$ uniformly. Since $B_{p s}^{-1}$ is $L^{2}$ bounded, by (2.37) we have

$$
\left\|B_{p s}^{-1} \tilde{P}_{p s} B_{p s} f\right\|_{L^{2} L^{2}} \lesssim\left\|\tilde{P}_{p s} B_{p s} f\right\|_{L^{2} L^{2}} \lesssim\left\|B_{p s} f\right\|_{L^{p_{3}^{\prime} H^{\rho_{3}, q_{3}^{\prime}}}} \lesssim\|f\|_{L^{p_{2}^{\prime} H^{\rho_{2}, q_{2}^{\prime}}}}
$$

and

$$
\begin{aligned}
& \left\|\partial_{t} B_{p s}^{-1} \tilde{P}_{p s} B_{p s} f\right\|_{L^{2} L^{2}} \lesssim\left\|\left[\partial_{t}, B_{p s}^{-1}\right] \tilde{P}_{p s} B_{p s} f\right\|_{L^{2} L^{2}}+\left\|B_{p s}^{-1} \partial_{t} \tilde{P}_{p s} B_{p s} f\right\|_{L^{2} L^{2}}
\end{aligned}
$$

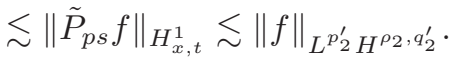

By Sobolev embeddings we obtain the desired bound.

It remains to consider the error estimate,

$$
\left\|L_{K} P_{p s} f-f\right\|_{L E K_{p s}^{*}+L^{1} L^{2}} \lesssim\|f\|_{L_{2}^{p_{2}^{\prime} \rho_{2}, q_{2}^{\prime}}},
$$

for which we compute

$$
\begin{aligned}
L_{K} P_{p s} f-f= & {\left[L_{K}, B_{p s}^{-1}\right] \tilde{P}_{p s} B_{p s} f } \\
& +B_{p s}^{-1}\left(L_{K} \tilde{P}_{p s}-I\right) B_{p s} f \\
& +\left(B_{p s}^{-1} B_{p s}-I\right) f .
\end{aligned}
$$

We consider each term in the above decomposition. For the first term, due to the $H_{t, x}^{1}$ bound for $\tilde{P}_{p s}$ in (2.37), we need the commutator bound

$$
\left[L_{K}, B_{p s}^{-1}\right]: H_{t, x}^{1} \rightarrow L E K_{p s}^{*},
$$

or equivalently

$$
B_{p s}\left[L_{K}, B_{p s}^{-1}\right]: H_{t, x}^{1} \rightarrow L^{2} L^{2}
$$

which is almost identical to (2.32) and is proved in the same manner.

The bound for the second term is a direct consequence of the $L^{2} L^{2}$ error bound for $\tilde{P}_{p s}$.

Finally, for the last term we know that $\left(B_{p s}^{-1} B_{p s}-I\right) \in O P S_{1-\delta, 0}^{-1+\delta}$; therefore using Sobolev embeddings and the properties of pseudodifferential operators we estimate

$$
\left\|\left(B_{p s}^{-1} B_{p s}-I\right) f\right\|_{L^{p_{2}^{\prime}} H^{\frac{1}{2}}} \lesssim\|f\|_{L^{p_{2}^{\prime} H^{\rho_{2}, q_{2}^{\prime}}} .} .
$$

This concludes the proof of (2.36) since

$$
L^{p_{2}^{\prime}} H^{\frac{1}{2}} \subset L^{2} H^{\frac{1}{2}}+L^{1} H^{\frac{1}{2}} \subset L E K_{p s}^{*}+L^{1} L^{2} .
$$

\section{ACKNOWLEDGEMENTS}

The author would like to thank Daniel Tataru for many useful conversations and suggestions regarding the paper. 


\section{REFERENCES}

[1] L. Andersson and P. Blue: Hidden symmetries and decay for the wave equation on the Kerr spacetime, arXiv:0908.2265v1

[2] P. Brenner: On $L_{p}-L_{p^{\prime}}$ estimates for the wave-equation. Math. Z. 145 (1975), 251-254. MR0387819(52:8658)

[3] S. Chandrasekhar: The Mathematical Theory of Black Holes. Oxford Classic Texts in the Physical Sciences. Oxford University Press, 1992 MR 1210321 (93k:83046)

[4] M. Dafermos and I. Rodnianski: A proof of the uniform boundedness of solutions to the wave equation on slowly rotating Kerr backgrounds, arXiv:0805.4309v1

[5] M. Dafermos and I. Rodnianski: Lectures on black holes and linear waves, arXiv:0811.0354v1

[6] F. Finster, N. Kamran, J. Smoller, S. T. Yau: Decay of solutions of the wave equation in Kerr geometry. Comm. Math. Phys. 264 (2006), 465-503. MR2215614(2007b:83019)

[7] F. Finster, J. Smoller: A Time Independent Energy Estimate for Outgoing Scalar Waves in the Kerr Geometry. J. Hyperbolic Differ. Equ. 5 (2008), no. 1, 221-255. MR2405857 (2009d:35330)

[8] J. Ginibre and G. Velo: Generalized Strichartz inequalities for the wave equation. J. Funct. Anal. 133 (1995), 50-68. MR.1351643 (97a:46047)

[9] S. W. Hawking and G. F. R. Ellis: The large scale structure of space-time. Cambridge Monographs on Mathematical Physics, No. 1. London, New York: Cambridge University Press, 1973. MR0424186(54:12154)

[10] L. Kapitanski: Some generalizations of the Strichartz-Brenner inequality. Leningrad Math. J. 1 (1990), 693-726. MR 1015129 (90h:46063)

[11] M. Keel and T. Tao: Endpoint Strichartz estimates. Amer. J. Math. 120 (1998), 955-980. MR1646048 (2000d:35018)

[12] H. Lindblad and C. D. Sogge: On existence and scattering with minimal regularity for semilinear wave equations. J. Funct. Anal. 130 (1995), 357-426. MR1335386 (96i:35087)

[13] J. Marzuola, J. Metcalfe, D. Tataru, and M. Tohaneanu: Strichartz estimates on Schwarzschild black hole backgrounds. Comm. Math. Phys. 293 (2010), no. 1, 37-83. MR2563798 (2010m:58043)

[14] J. Metcalfe and D. Tataru: Global parametrices and dispersive estimates for variable coeffcient wave equations, arXiv:0707.1191.

[15] G. Mockenhaupt, A. Seeger, and C. D. Sogge: Local smoothing of Fourier integral operators and Carleson-Sjölin estimates. J. Amer. Math. Soc. 6 (1993), 65-130. MR1168960 (93h:58150)

[16] H. Pecher: Nonlinear small data scattering for the wave and Klein-Gordon equations. Math. Z. 185 (1984), 261-270. MR.731347 (85h:35165)

[17] H. F. Smith: A parametrix construction for wave equations with $C^{1,1}$ coefficients. Ann. Inst. Fourier (Grenoble) 48 (1998), 797-835. MR1644105 (99h:35119)

[18] R. S. Strichartz: A priori estimates for the wave equation and some applications. J. Funct. Anal. 5 (1970), 218-235. MR0257581 (41:2231)

[19] R. S. Strichartz: Restrictions of Fourier transforms to quadratic surfaces and decay of solutions of wave equations. Duke Math. J. 44 (1977), 705-714. MR.0512086 (58:23577)

[20] D. Tataru: Strichartz estimates for operators with nonsmooth coefficients and the nonlinear wave equation. Amer. J. Math. 122 (2000), 349-376. MR:1749052 (2001c:35260)

[21] D. Tataru: Strichartz estimates for second order hyperbolic operators with nonsmooth coefficients II. Amer. J. Math. 123 (2001), 385-423. MR1833146 (2002d:35140)

[22] D. Tataru: Strichartz estimates for second order hyperbolic operators with nonsmooth coefficients III. J. Amer. Math. Soc. 15 (2002), 419-442. MR1887639(2003a:35120)

[23] D. Tataru and M. Tohaneanu: Local energy estimate on Kerr black hole backgrounds, arXiv:0810.5766v2

Department of Mathematics, Purdue University, West Lafayette, Indiana 47907-2067

Current address: Department of Mathematics, Johns Hopkins University, Baltimore, Maryland 21218 